Participant ID: 1 , Height $173 \mathrm{~cm}$, Weight $60 \mathrm{~kg}$, BMI $20 \mathrm{~kg} / \mathrm{cm}$, age: $20 \mathrm{~s}$, sex: male

\title{
walking CPR
}

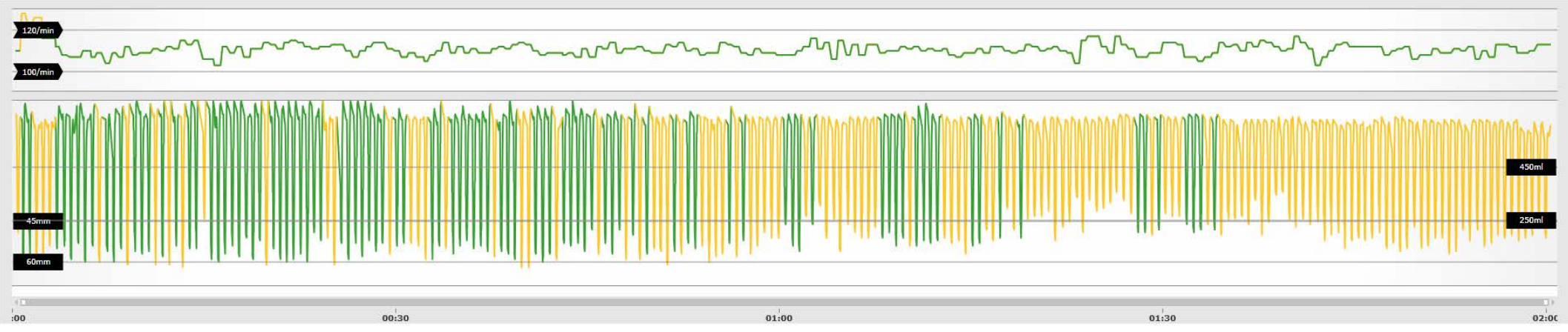

\section{straddling CPR}

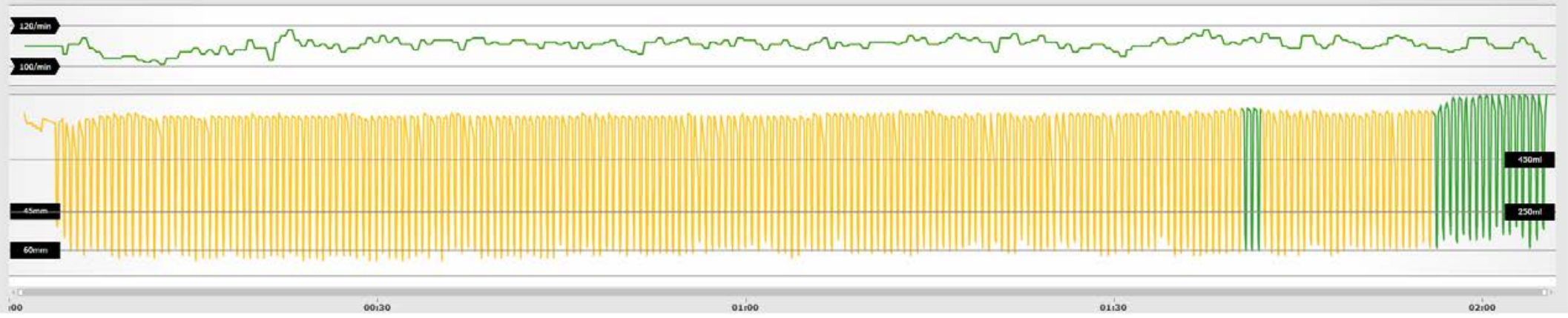

pretest

음

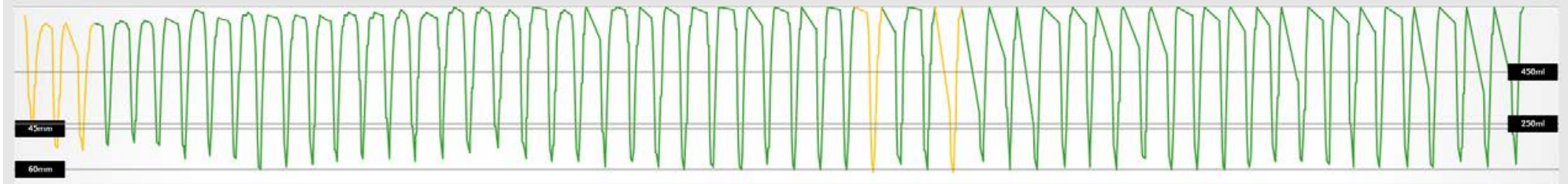

io 
Participant ID: 2, Height $166 \mathrm{~cm}$, Weight $66 \mathrm{~kg}$, BMI $24 \mathrm{~kg} / \mathrm{cm}$, age: $30 \mathrm{~s}$, sex: male

walking CPR

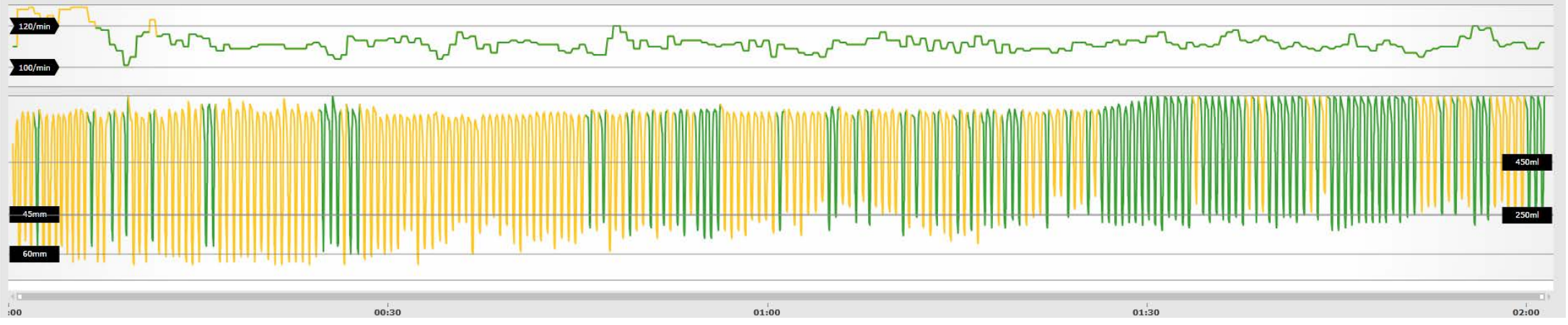

straddling CPR

$120 / \min$

$200 / \min$

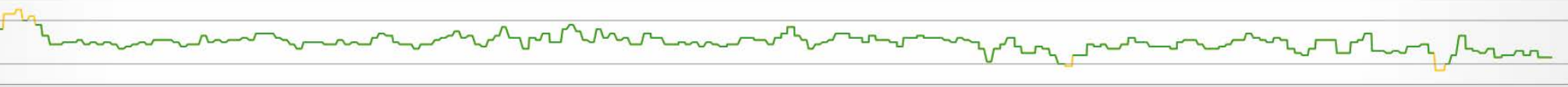

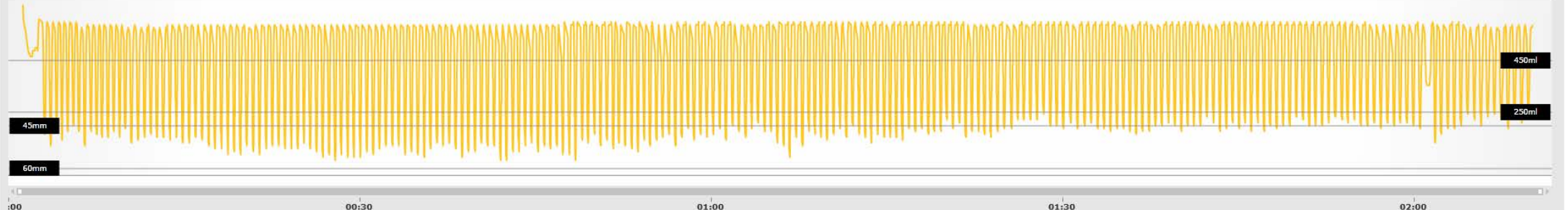

(1)

01:00

02:00

pretest

100/min

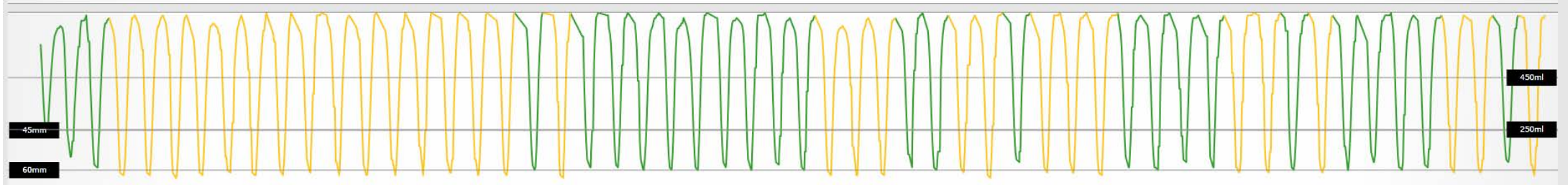


Participant ID: 3, Height $155 \mathrm{~cm}$, Weight $47 \mathrm{~kg}$, BMI $20 \mathrm{~kg} / \mathrm{cm}$, age: $40 \mathrm{~s}$, sex: female

\section{walking CPR}

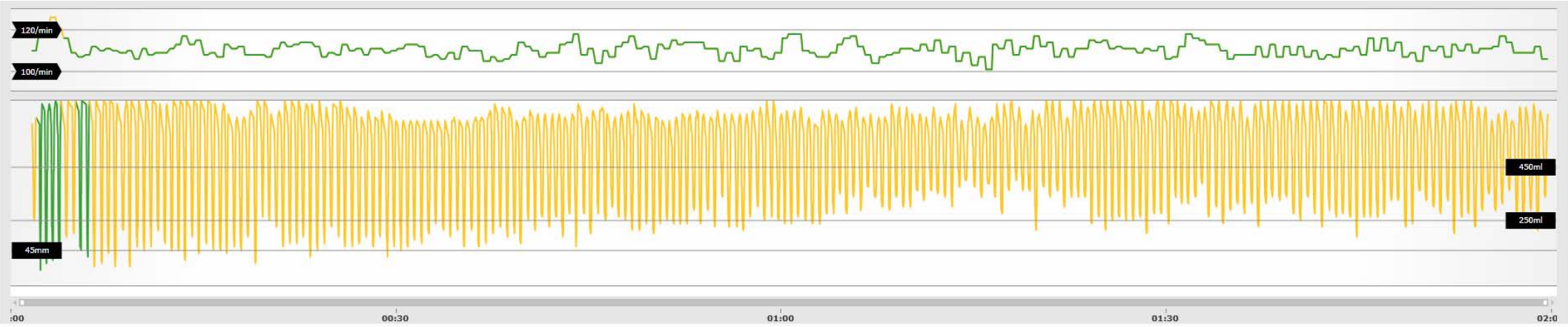

\section{straddling CPR}

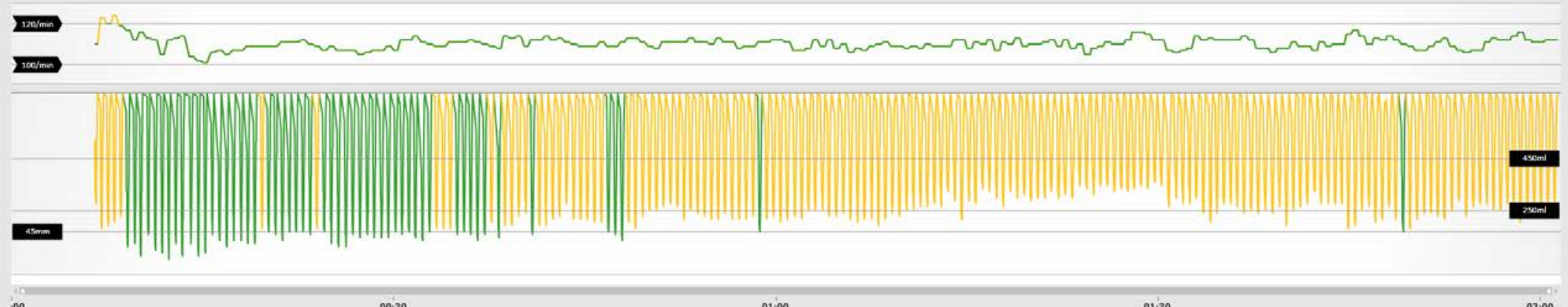

pretest

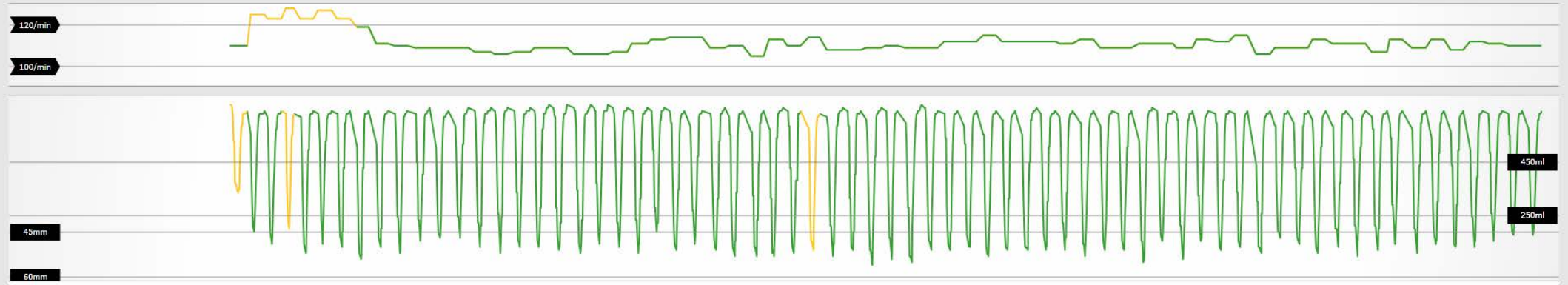

$60 \mathrm{~mm}$ 
Participant ID: 4, Height $150 \mathrm{~cm}$, Weight $58 \mathrm{~kg}$, BMI $26 \mathrm{~kg} / \mathrm{cm}$, age: $30 \mathrm{~s}$, sex: female

walking CPR

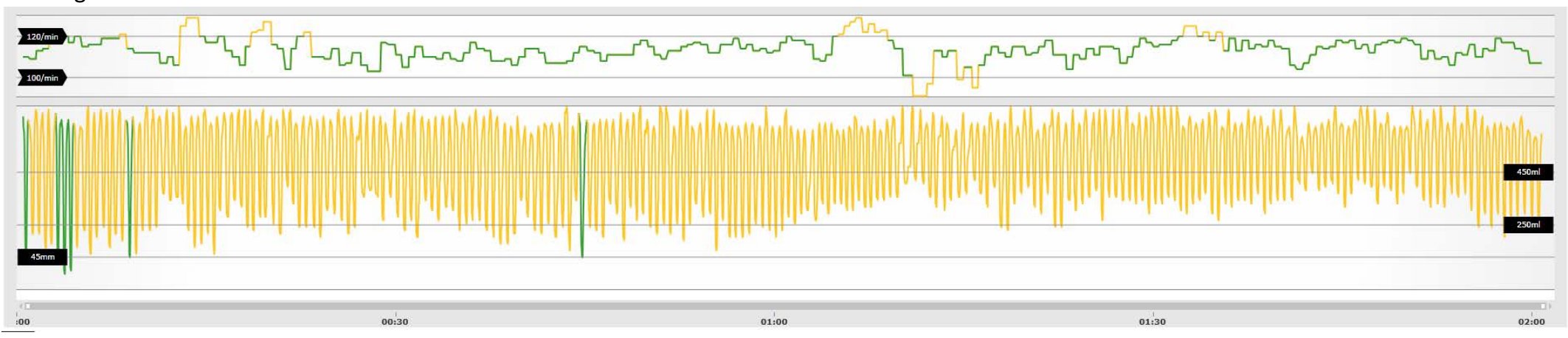

straddling CPR

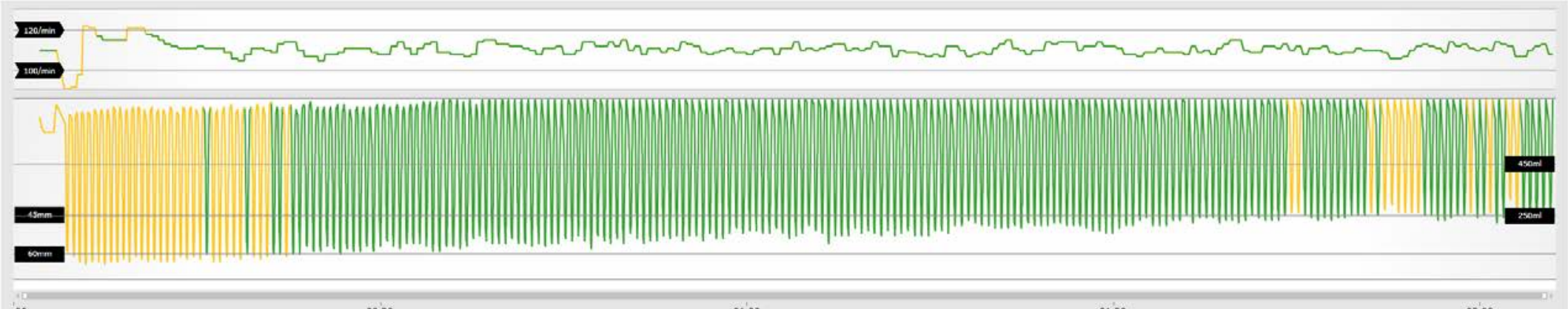

100 oniso

0100

01130

pretest

$\Xi$

(Asmm 
Participant ID: 5, Height $163 \mathrm{~cm}$, Weight $52 \mathrm{~kg}$, BMI $20 \mathrm{~kg} / \mathrm{cm}$, age: $30 \mathrm{~s}$, sex: female

walking CPR

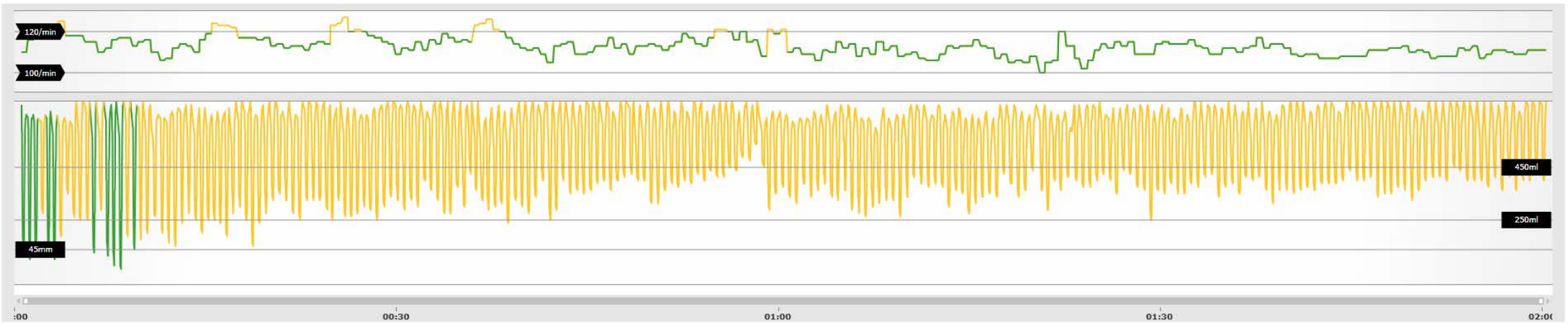

straddling CPR

-

100/min

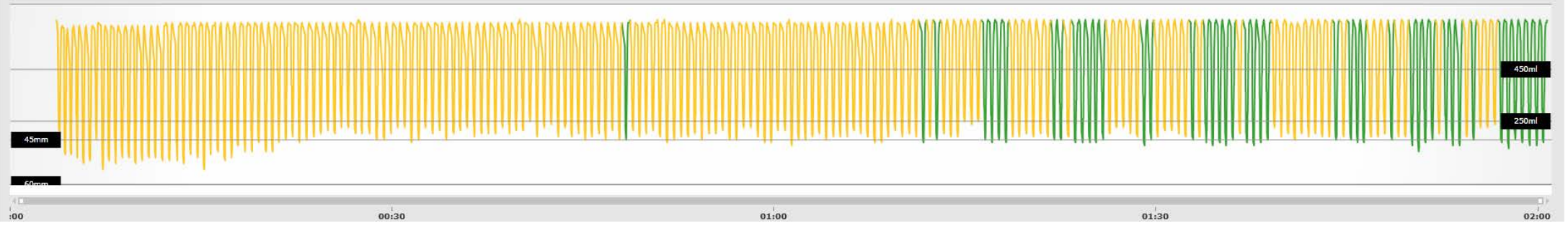

\section{pretest}

\section{E} $=$

, 
Participant ID: 6, Height $173 \mathrm{~cm}$, Weight $68 \mathrm{~kg}$, BMI $23 \mathrm{~kg} / \mathrm{cm}$, age: $20 \mathrm{~s}$, sex: male

\section{walking CPR}

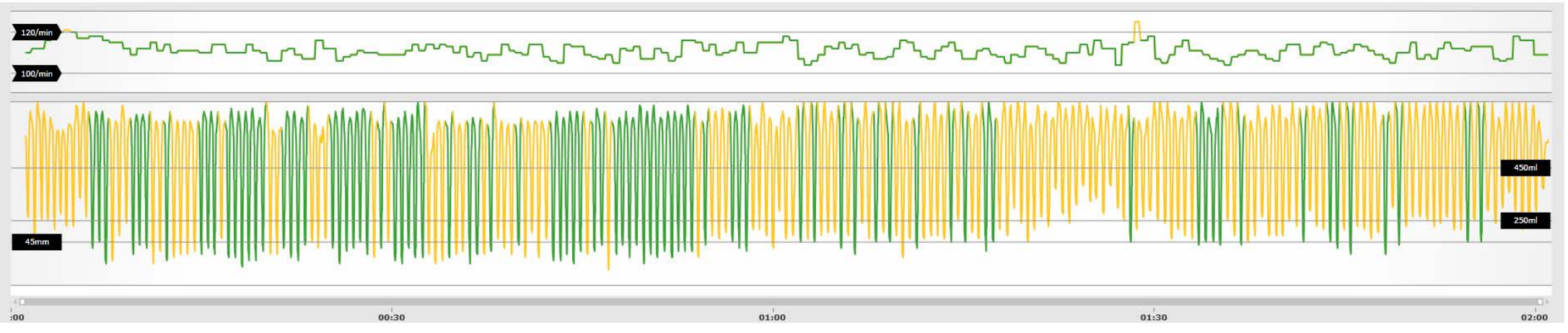

\section{straddling CPR}

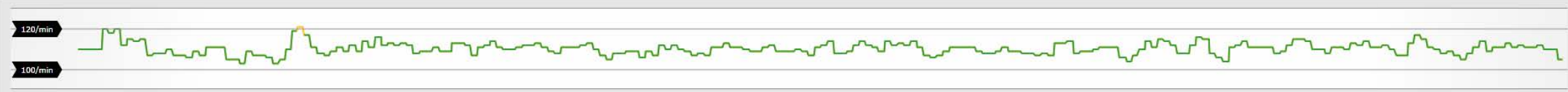

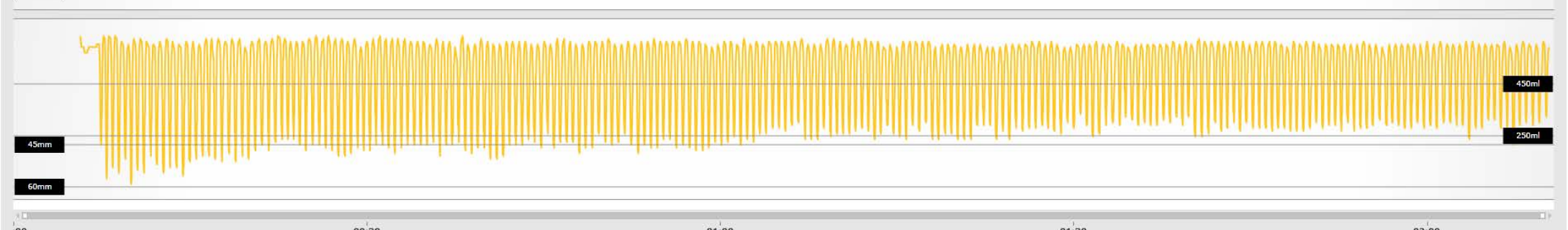

:00 00:30 $\quad$ 01:00 $02: 00$

pretest

$1200 /$ min

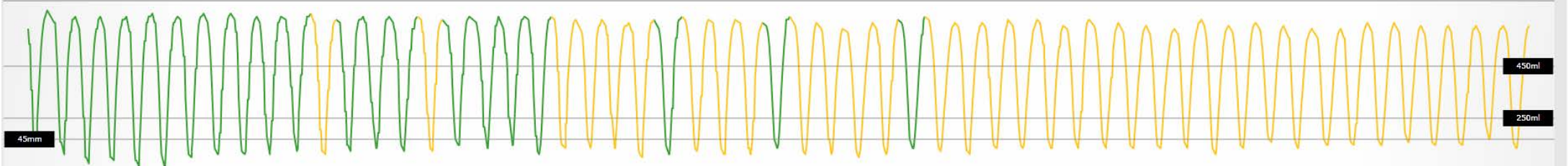

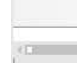


Participant ID: 7, Height $187 \mathrm{~cm}$, Weight $69 \mathrm{~kg}, \mathrm{BMI} 20 \mathrm{~kg} / \mathrm{cm}$, age: $40 \mathrm{~s}$, sex: male

walking CPR

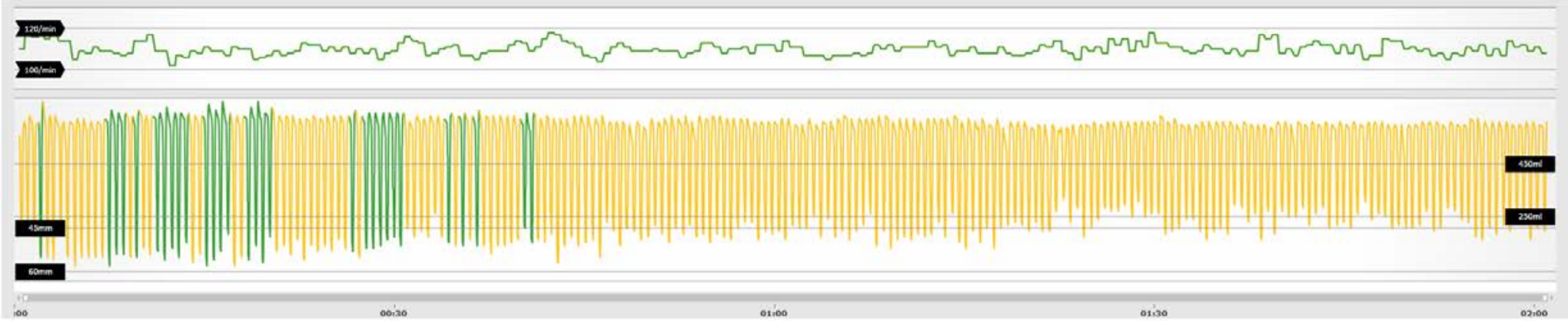

straddling CPR

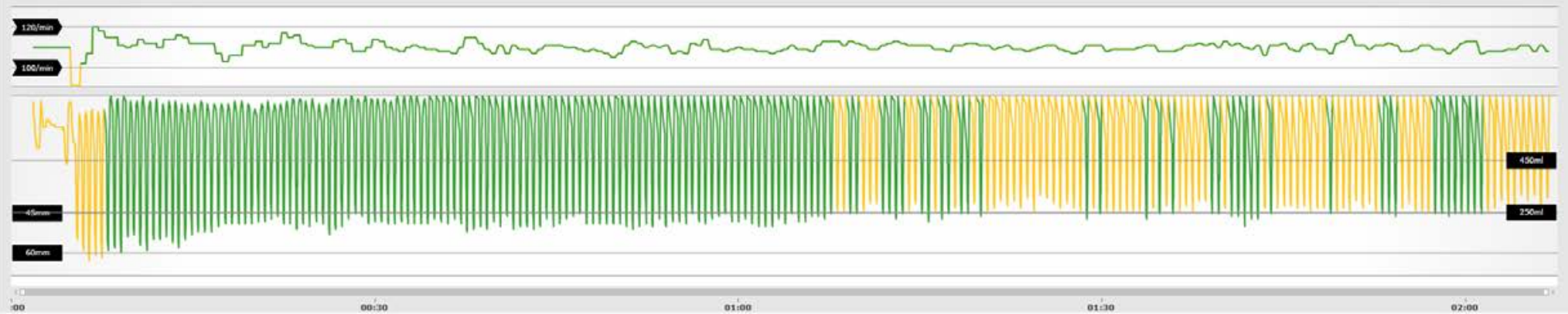

pretest

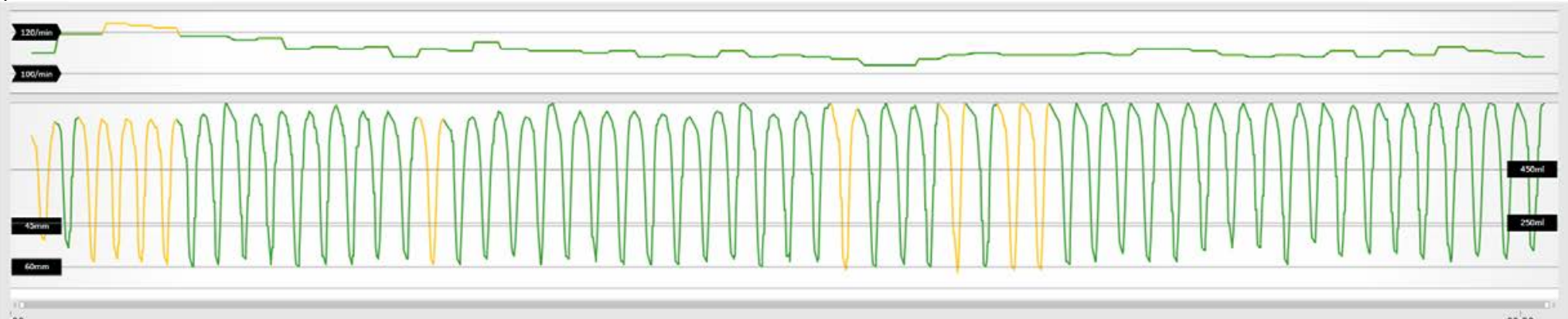


Participant ID: 8 , Height $159 \mathrm{~cm}$, Weight $56 \mathrm{~kg}$, BMI $22 \mathrm{~kg} / \mathrm{cm}$, age: $40 \mathrm{~s}$, sex: female

walking CPR

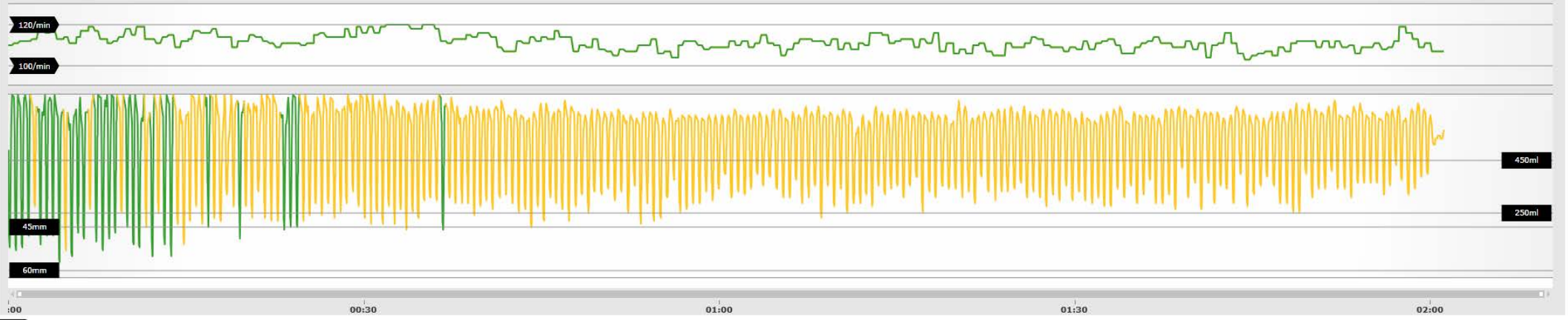

straddling CPR

$21200 / \min$

$100 /$ min

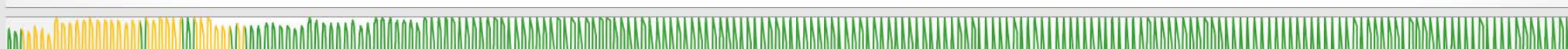

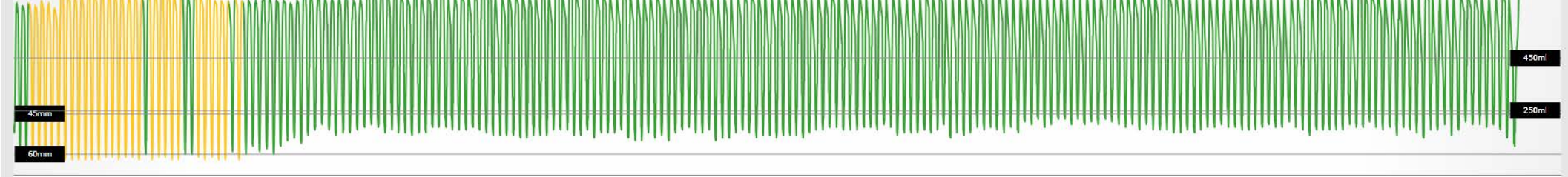

o0:30

01:30

02:00

pretest

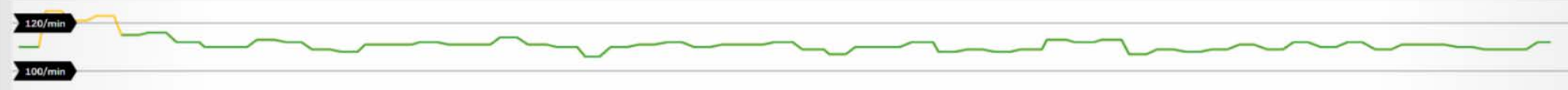

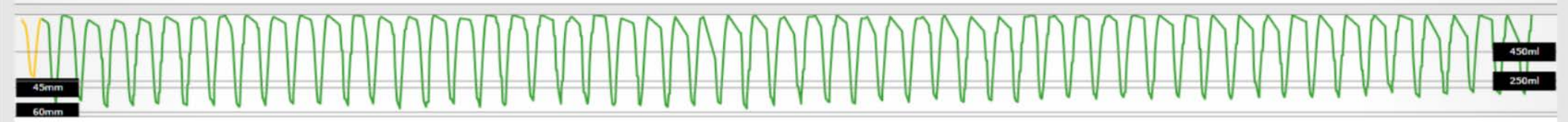

loo 
Participant ID: 9, Height $157 \mathrm{~cm}$, Weight $52 \mathrm{~kg}, \quad$ BMI $21 \mathrm{~kg} / \mathrm{cm}$, age: $30 \mathrm{~s}$, sex: female

\section{walking CPR}

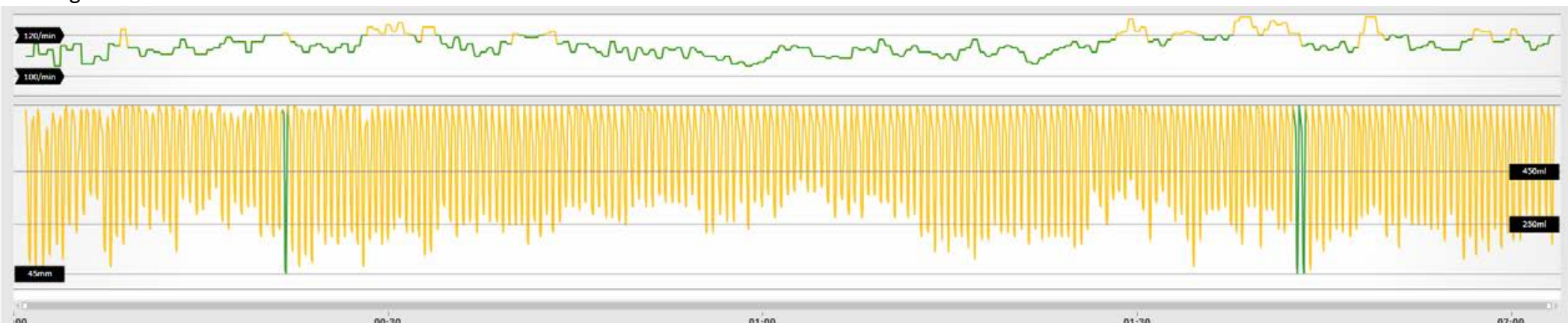

100

straddling CPR

$2 \mathrm{mogmim}$

I00/ming

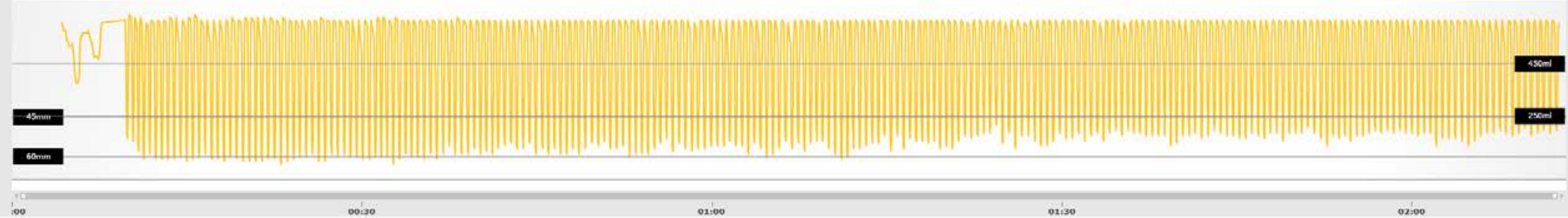

pretest

Iavm

100/min

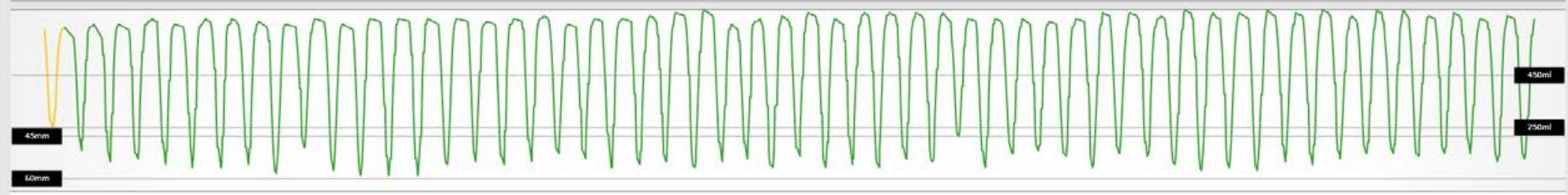


Participant ID: 10, Height $183 \mathrm{~cm}$, Weight $78 \mathrm{~kg}$, BMI $23 \mathrm{~kg} / \mathrm{cm}$, age: $20 \mathrm{~s}$, sex: male

\section{walking CPR}

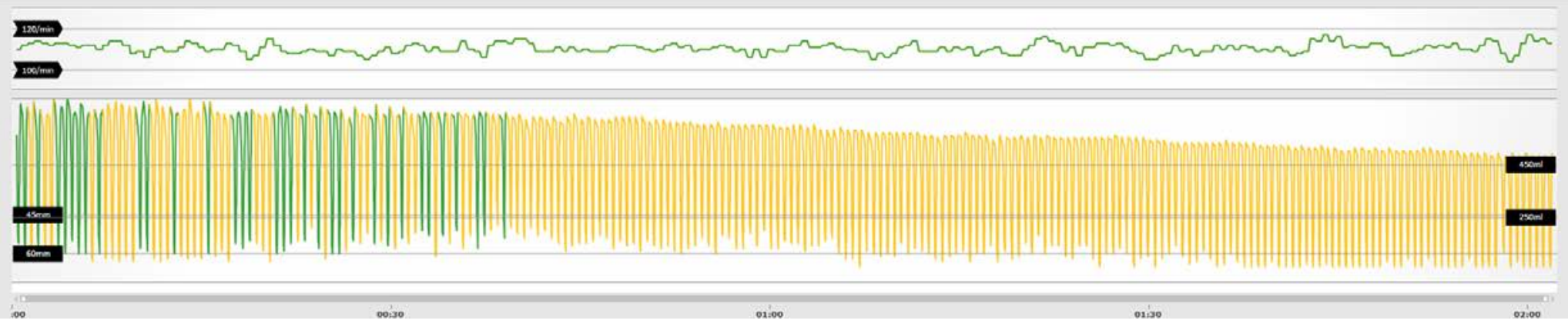

straddling CPR

(n)

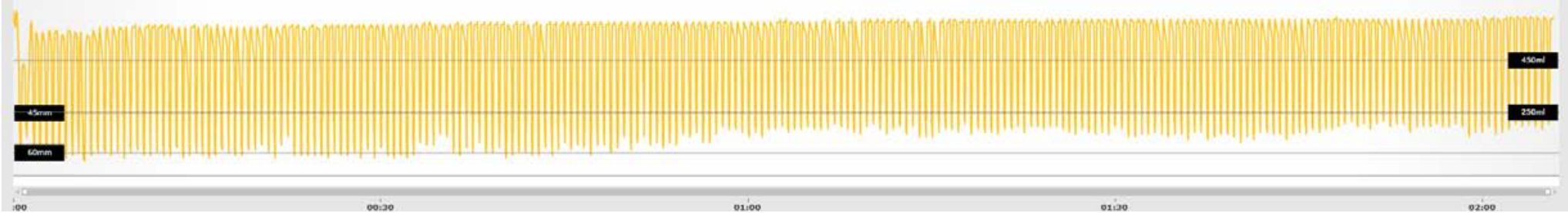

pretest

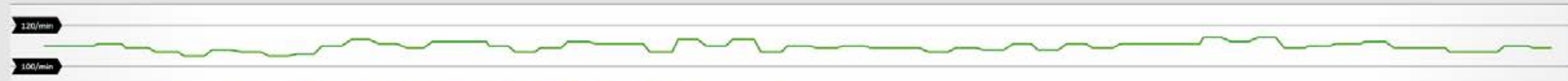

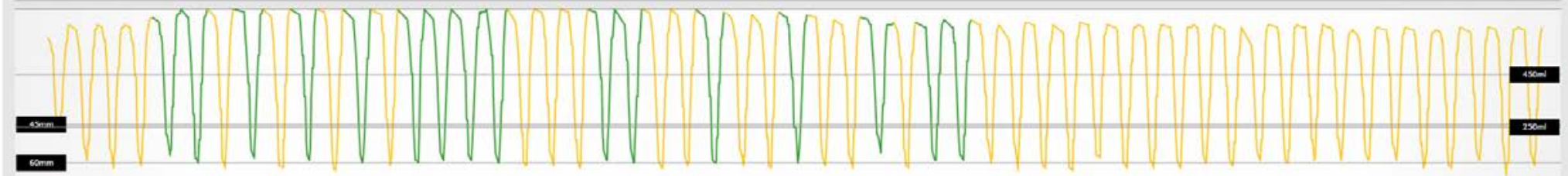


Participant ID: 11 , Height $167 \mathrm{~cm}$, Weight $60 \mathrm{~kg}$, BMI $22 \mathrm{~kg} / \mathrm{cm}$, age: $60 \mathrm{~s}$, sex: male

\section{walking CPR}

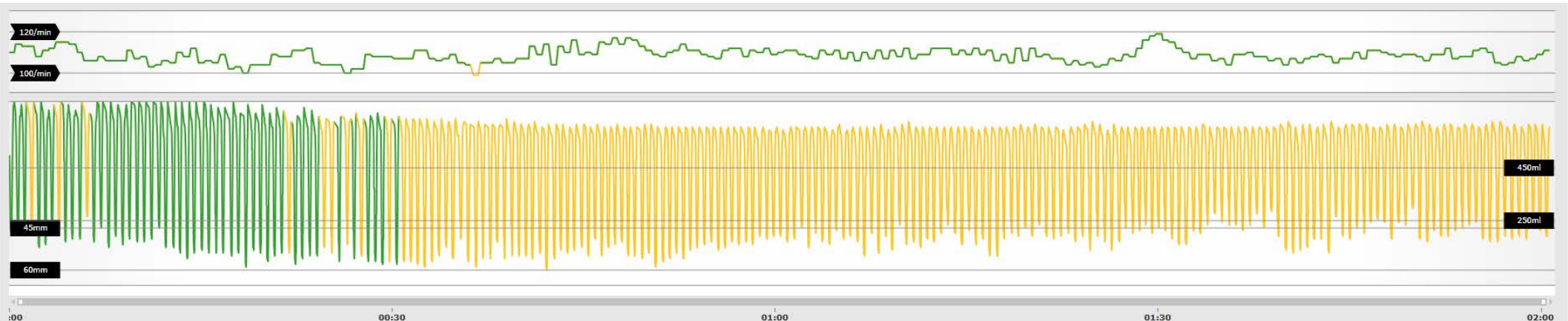

\section{straddling CPR}

120/min

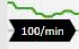

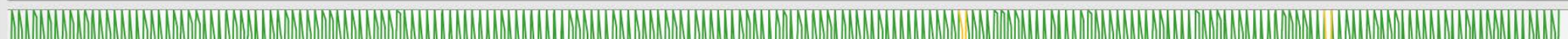

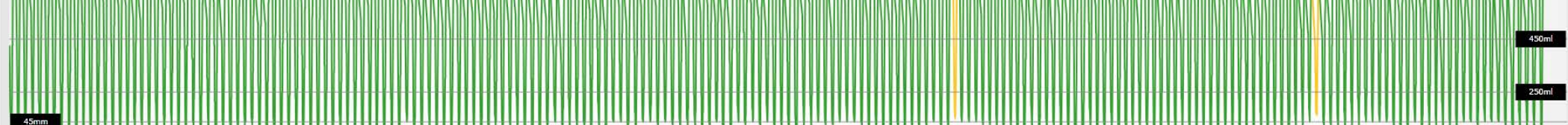

$\underset{45 m m}{1 . m}$

pretest

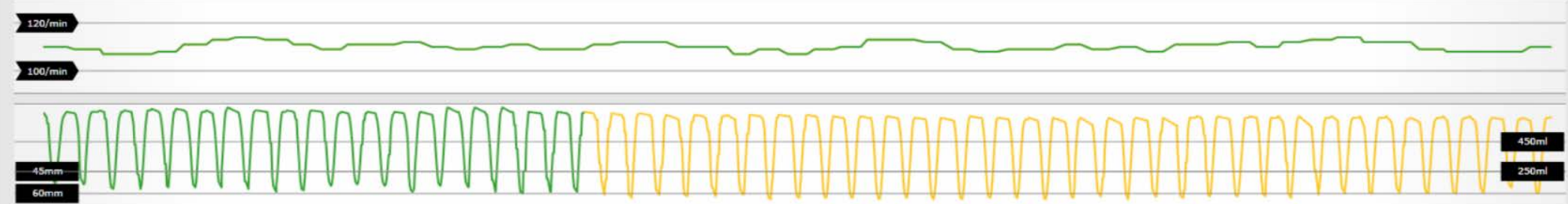


Participant ID: 12 , Height $165 \mathrm{~cm}$, Weight $58 \mathrm{~kg}, \quad$ BMI $21 \mathrm{~kg} / \mathrm{cm}$, age: $30 \mathrm{~s}$, sex: male

\section{walking CPR}

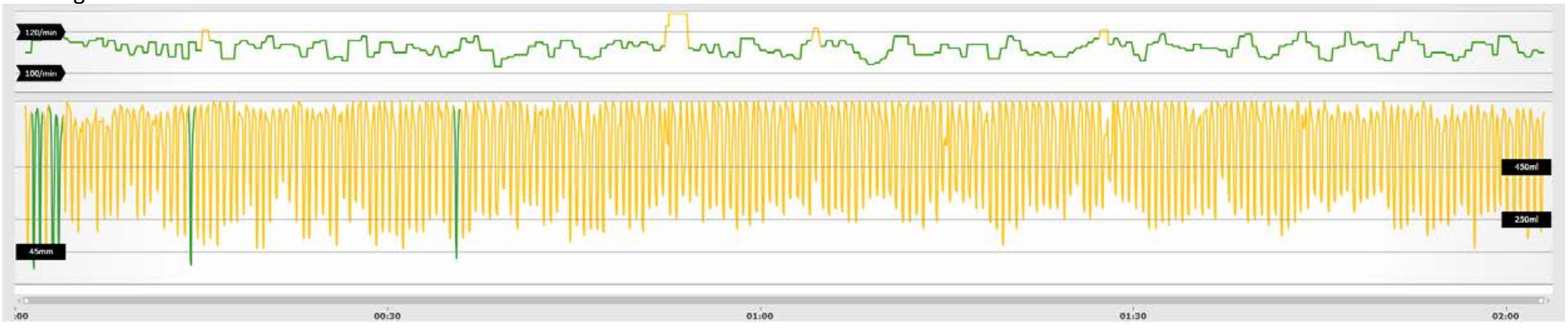

\section{straddling CPR}

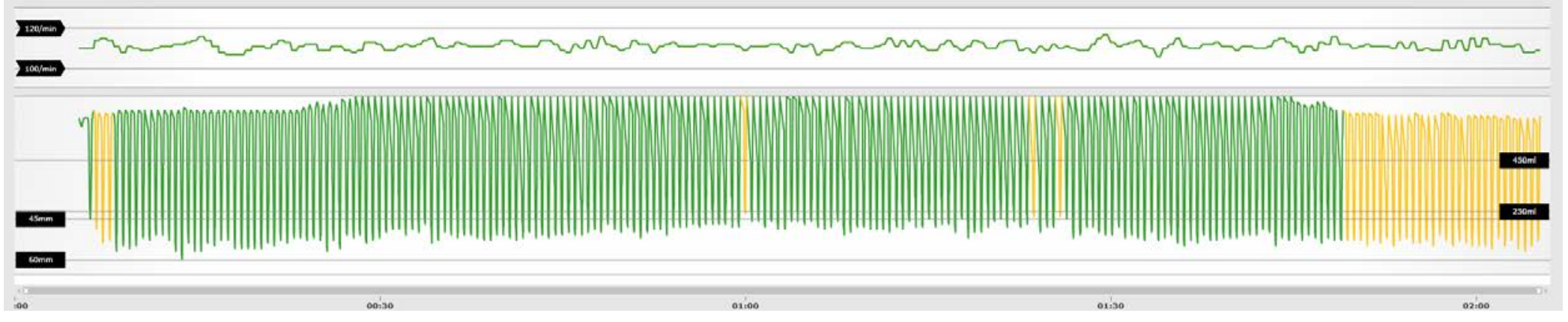

pretest

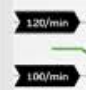

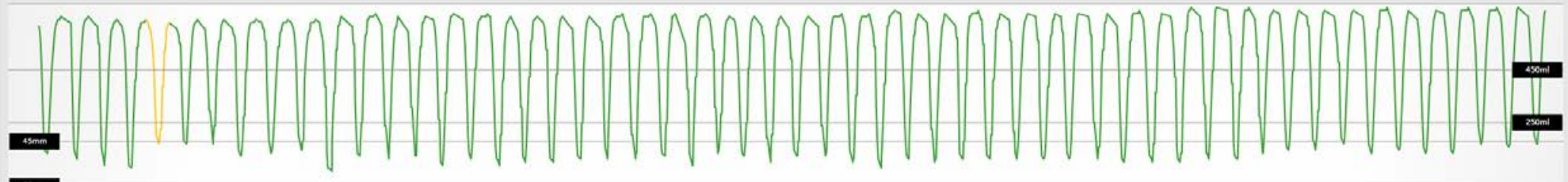

m.m. 
Participant ID: 13 , Height $159 \mathrm{~cm}$, Weight $52 \mathrm{~kg}, \mathrm{BMI} 21 \mathrm{~kg} / \mathrm{cm}$, age: $30 \mathrm{~s}$, sex: female

\section{walking CPR}

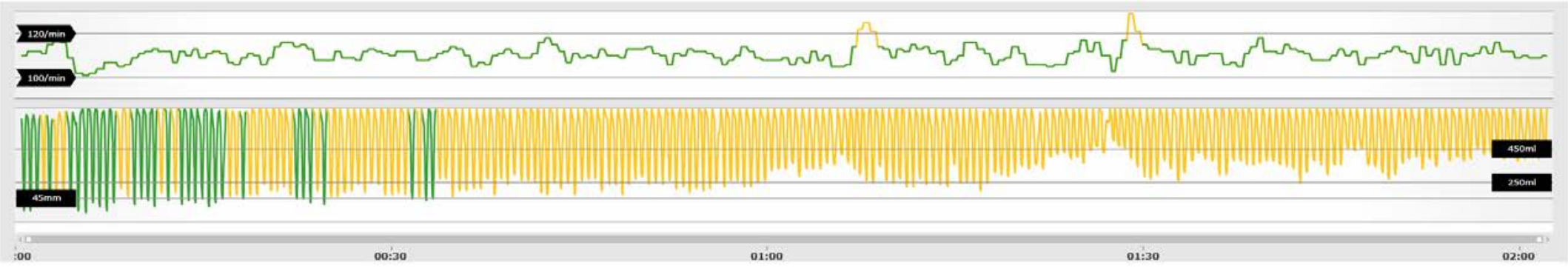

\section{straddling CPR}

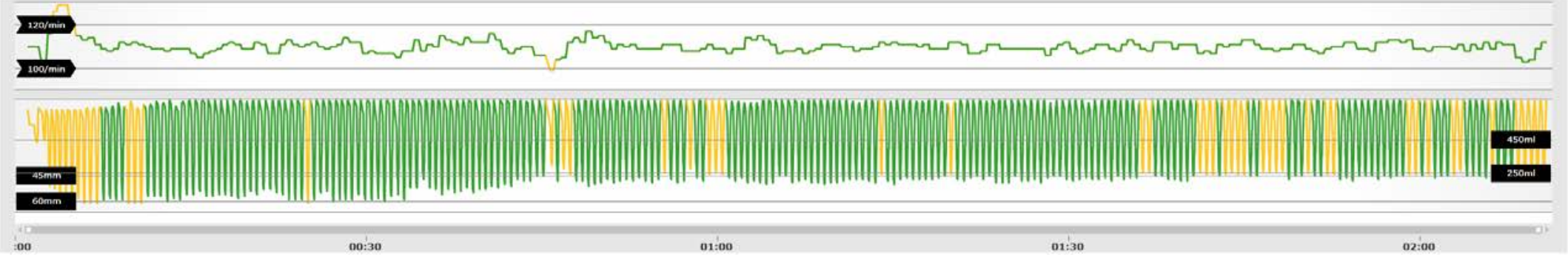

pretest

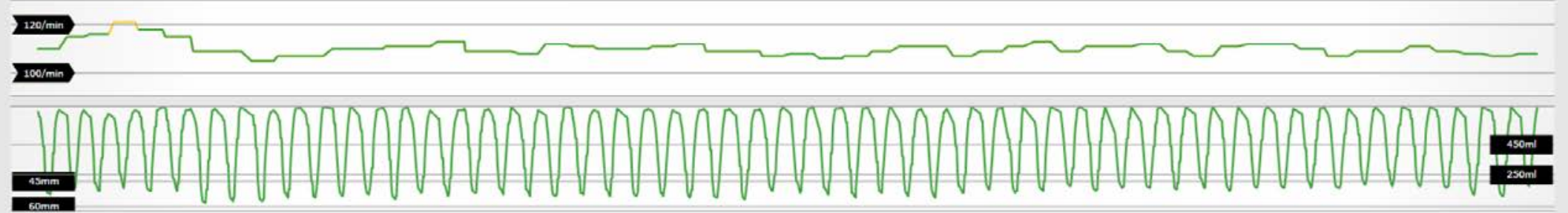


Participant ID: 14, Height $154 \mathrm{~cm}$, Weight $68 \mathrm{~kg}$, BMI $29 \mathrm{~kg} / \mathrm{cm}$, age: $40 \mathrm{~s}$, sex: female

\section{walking CPR}

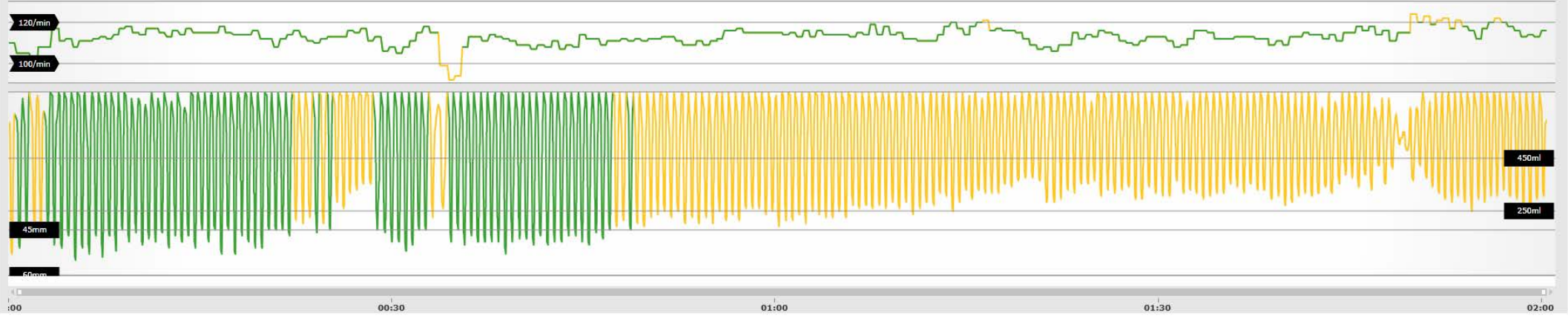

\section{straddling CPR}

I100/min

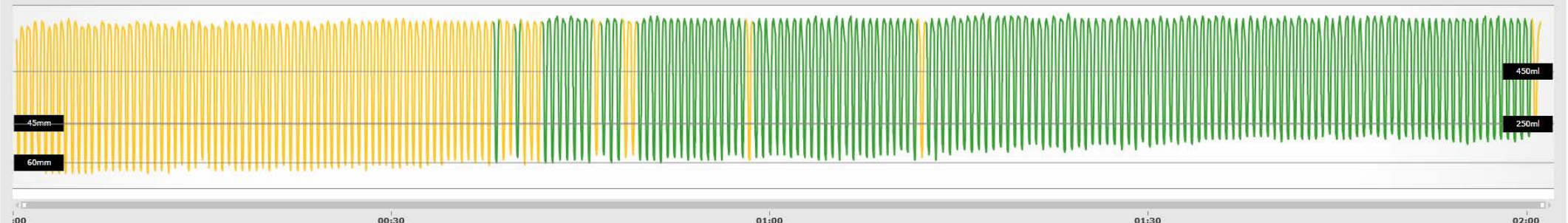

pretest

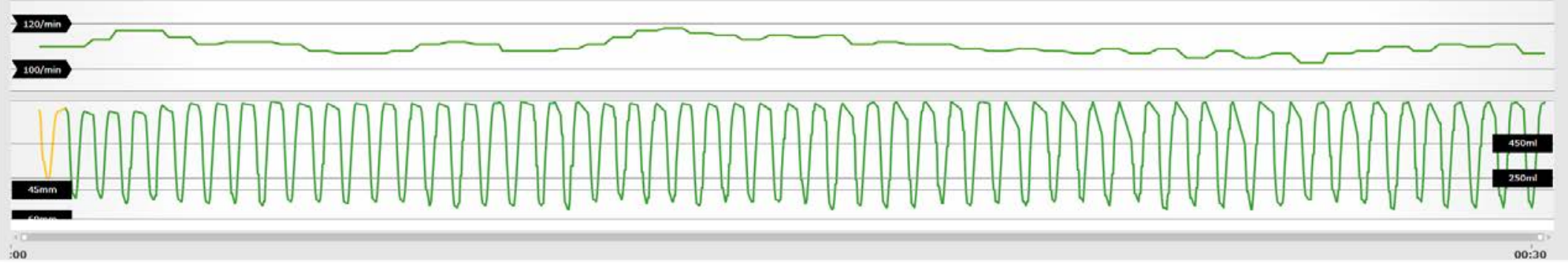


Participant ID: 15 , Height $174 \mathrm{~cm}$, Weight $74 \mathrm{~kg}, \mathrm{BMI} 24 \mathrm{~kg} / \mathrm{cm}$, age: $20 \mathrm{~s}$, sex: male

walking CPR

$\ln$

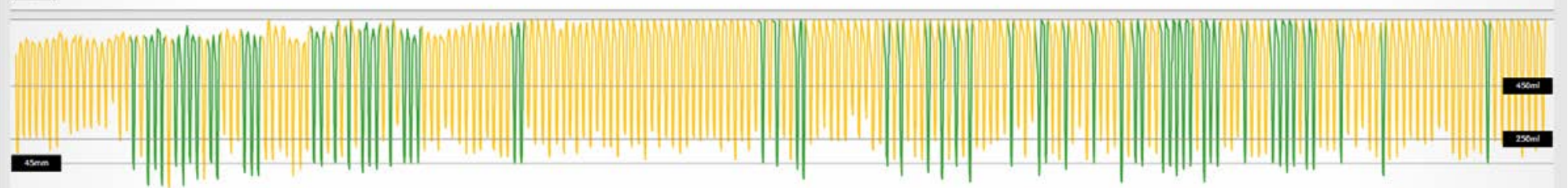

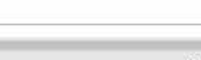

on.100

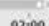

straddling CPR

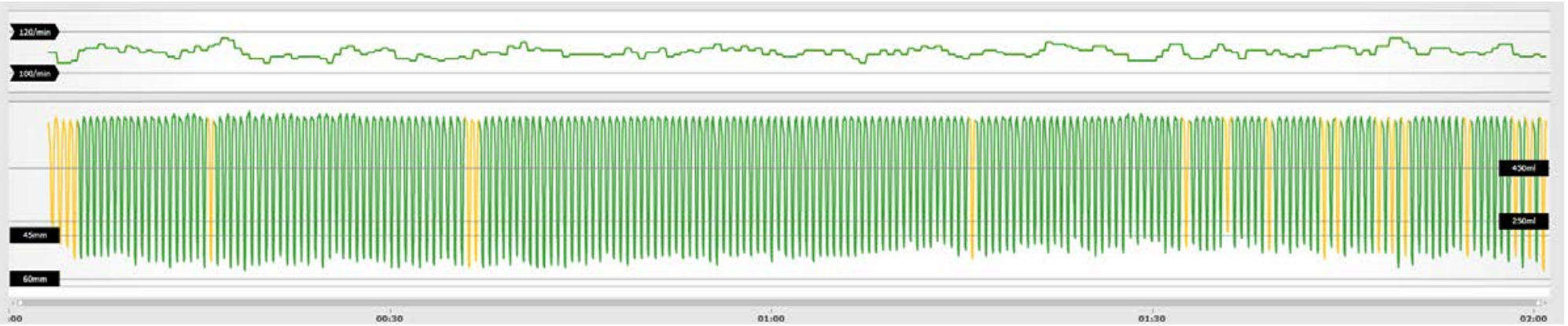

pretest

inimen

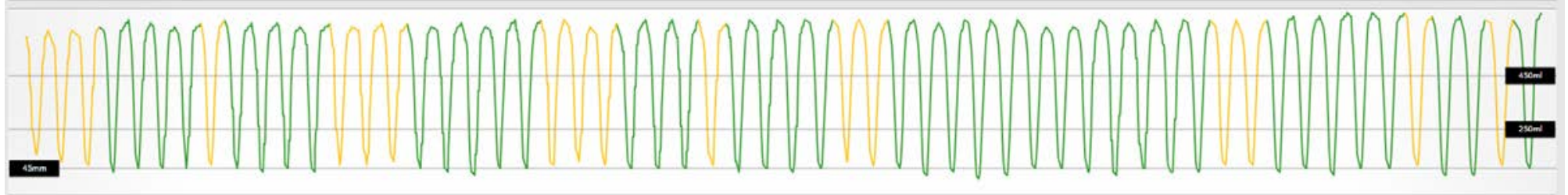

$\infty$ 
Participant ID: 16 , Height $162 \mathrm{~cm}$, Weight $67 \mathrm{~kg}$, BMI $26 \mathrm{~kg} / \mathrm{cm}$, age: $40 \mathrm{~s}$, sex: female

\section{walking CPR}

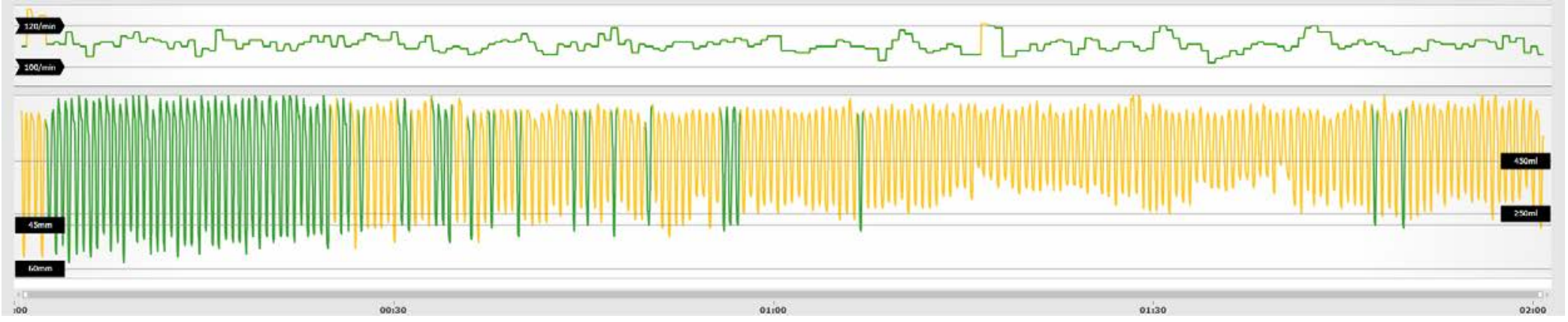

\section{straddling CPR}

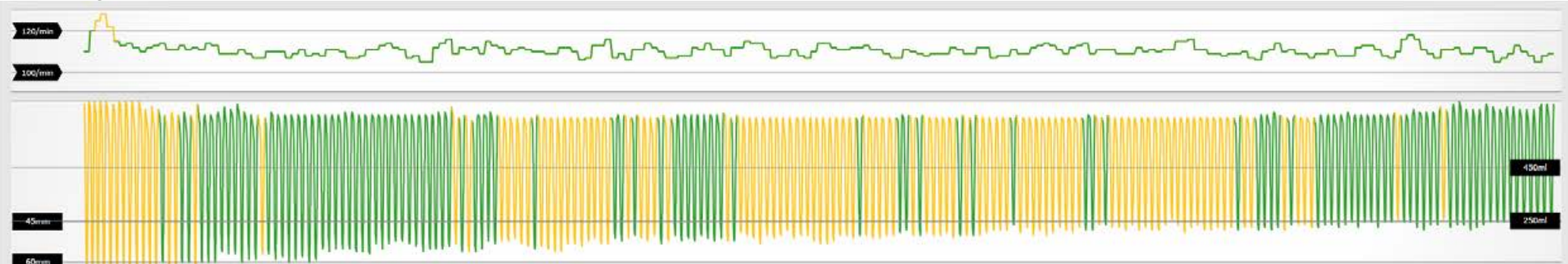

banm

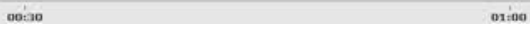

a1:30

I200/min

Inowm

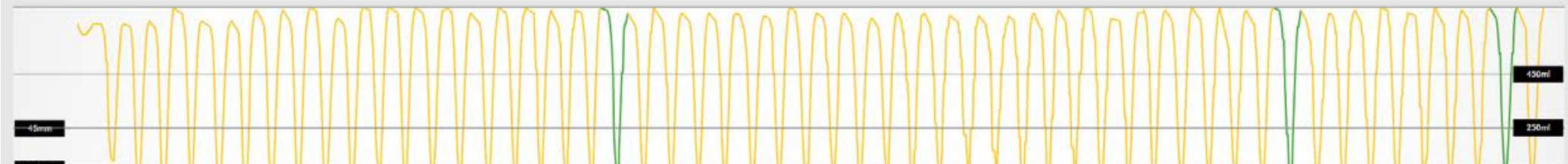

bamm 
Participant ID: 17 , Height $180 \mathrm{~cm}$, Weight $75 \mathrm{~kg}, \mathrm{BMI} 23 \mathrm{~kg} / \mathrm{cm}$, age: $30 \mathrm{~s}$, sex: male

walking CPR

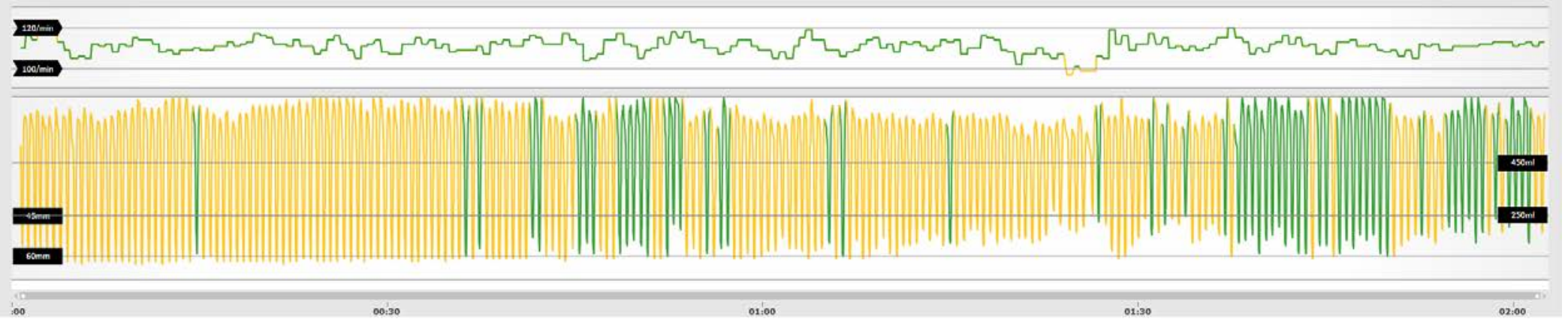

\section{straddling CPR}

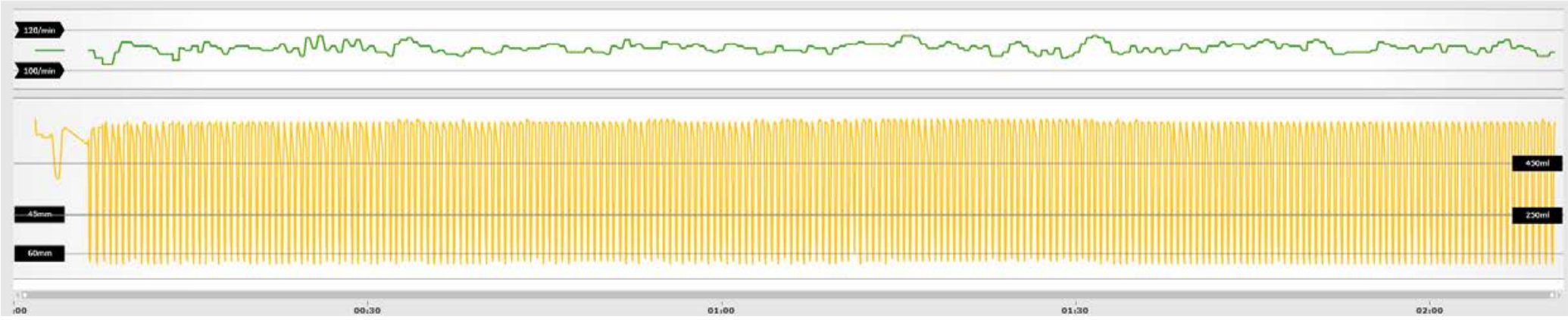

pretest

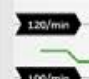

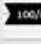

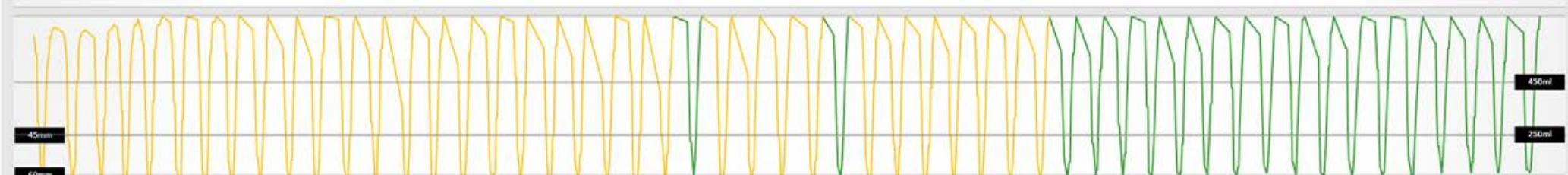

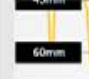

$\infty$ 
Participant ID: 18 , Height $158 \mathrm{~cm}$, Weight $62 \mathrm{~kg}, \mathrm{BMI} 25 \mathrm{~kg} / \mathrm{cm}$, age: $50 \mathrm{~s}$, sex: female

\section{walking CPR}

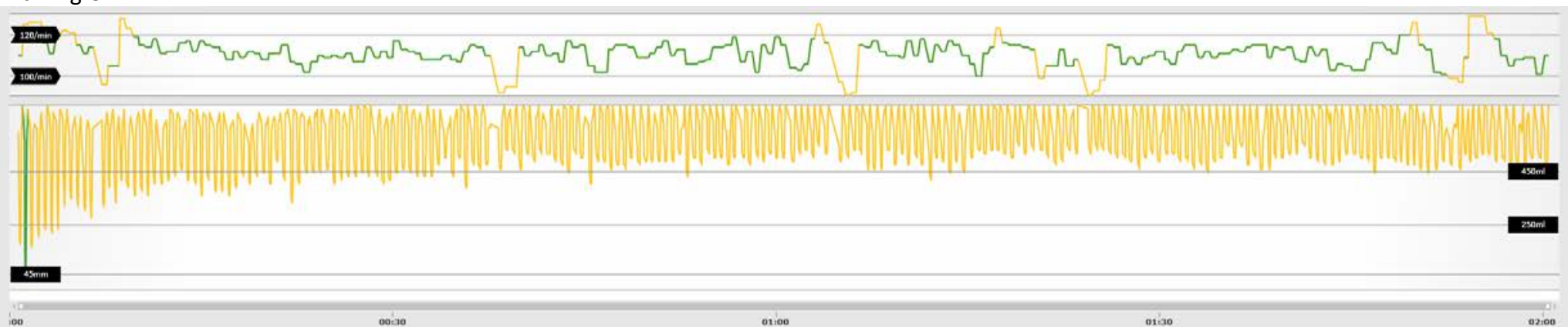

straddling CPR

Iromed

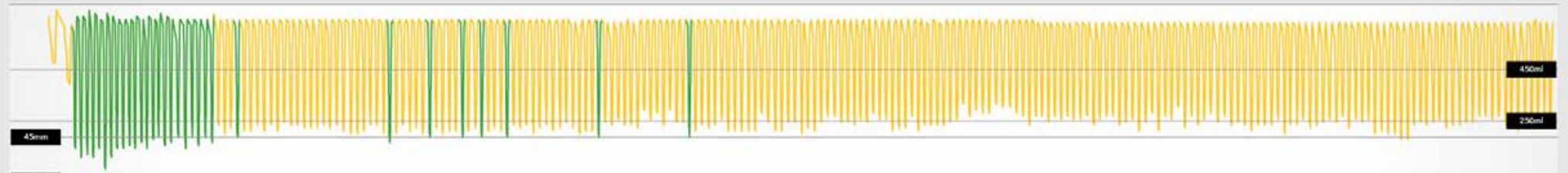

$+\cos$

oxioe

pretest

ind

Inc/ms

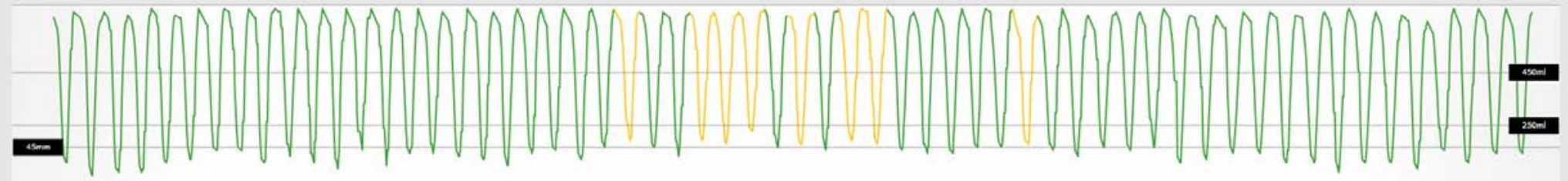


Participant ID: 19, Height $170 \mathrm{~cm}$, Weight $70 \mathrm{~kg}, \mathrm{BMI} 24 \mathrm{~kg} / \mathrm{cm}$, age: $20 \mathrm{~s}$, sex: male

walking CPR

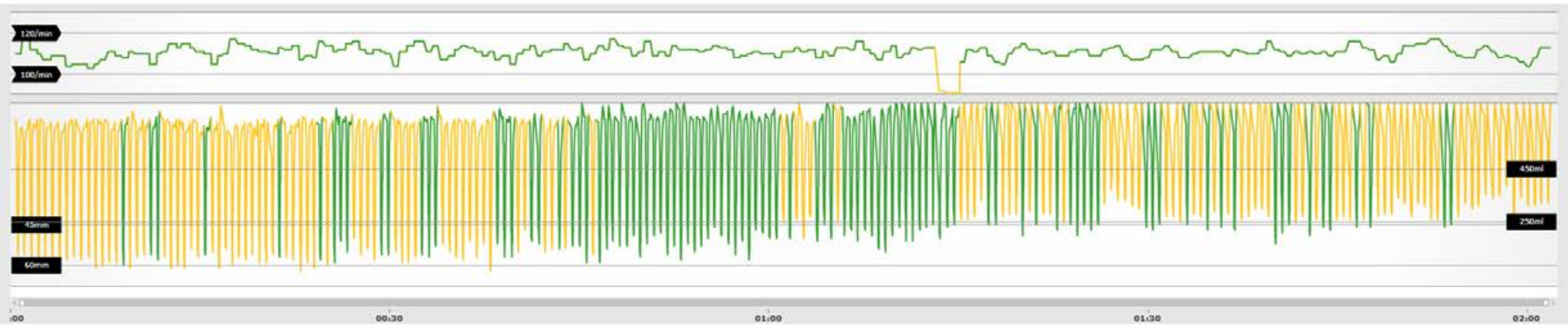

straddling CPR

Iraim

-

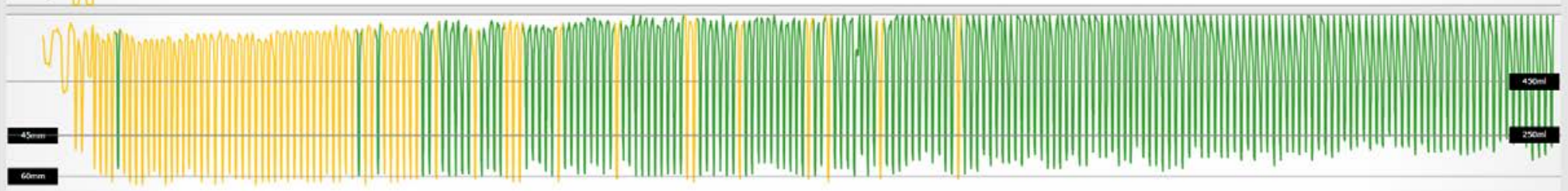
oei30 (1) 2200

pretest

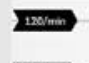

(2)

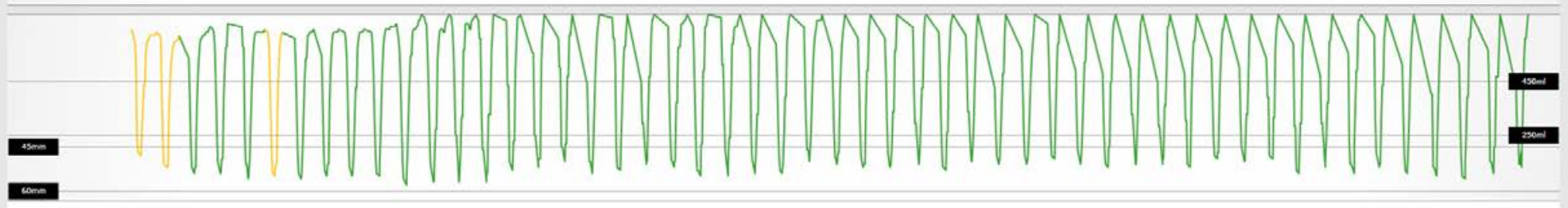


Participant ID: 20, Height $155 \mathrm{~cm}$, Weight $68 \mathrm{~kg}, \mathrm{BMI} 28 \mathrm{~kg} / \mathrm{cm}$, age: $30 \mathrm{~s}$, sex: female

\section{walking CPR}

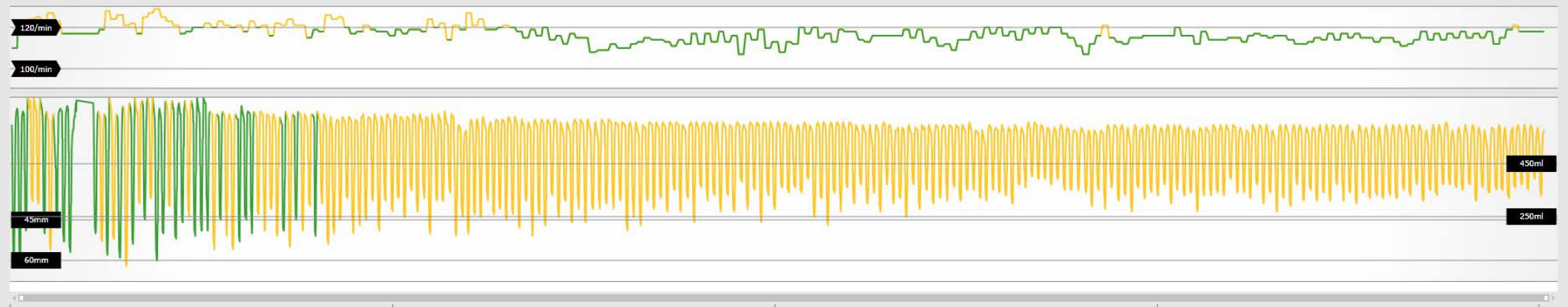

in

o0:30

a1:0o

$01: 30$

\section{straddling CPR}

$\sum_{100 / \mathrm{min}}^{200 / \mathrm{min}}$
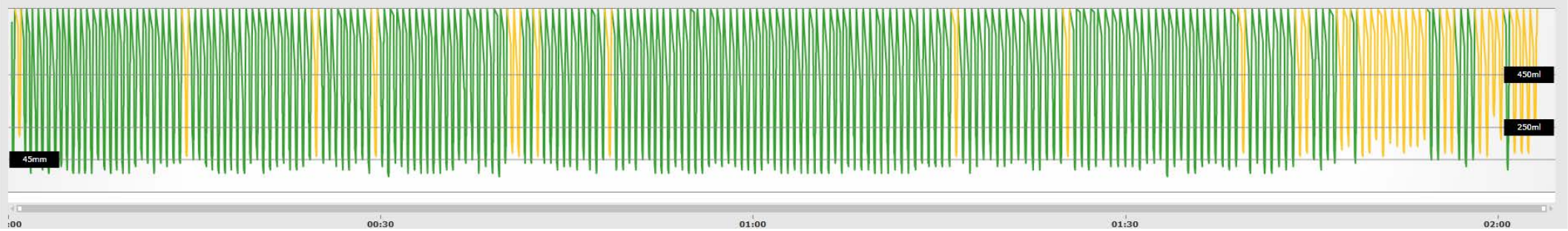

pretest

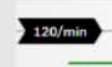

$100 / \min$

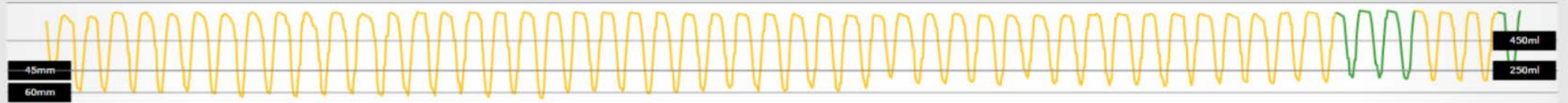


Participant ID: 21 , Height $170 \mathrm{~cm}$, Weight $55 \mathrm{~kg}$, BMI $19 \mathrm{~kg} / \mathrm{cm}$, age: $40 \mathrm{~s}$, sex: female excluded as a result of pretest

\section{pretest}

\section{0/min}
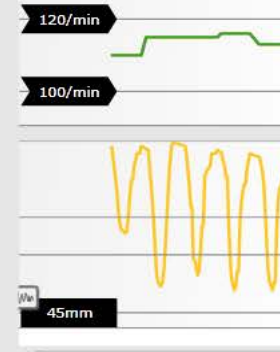

(2)

2

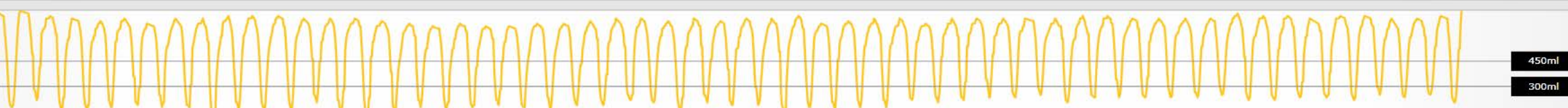


Participant ID: 22, Height $155 \mathrm{~cm}$, Weight $45 \mathrm{~kg}$, BMI $19 \mathrm{~kg} / \mathrm{cm}$, age: $20 \mathrm{~s}$, sex: female excluded as a result of pretest

\section{pretest}

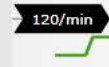

$100 /$ min

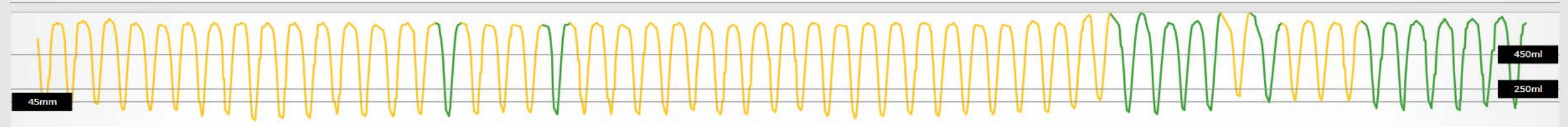

